\title{
Hacia una comprensión de las aproximaciones institucionales a los estudiantes con capacidades y talentos excepcionales: primeros pasos para incluirlos en la escuela
}

Understanding Institutional Approaches to Students with Exceptional Skills and TalentsFirst Steps to Include them in School

Para uma compreensão das abordagens institucionais para alunos com habilidades e talentos excepcionais: primeiros passos para incluí-los na escola

Maria Caridad García-Cepero* iD orcid.org/0000-0001-6064-6581 Juliana Iglesias-Velasco** (iD) orcid.org/0000-0002-3984-2905

Para citar este artículo: Garcia-Cepero, M.C., I glesias-Velasco, J. (2O2O). Comprendiendo las aproximaciones institucionales a los estudiantes con capacidades y talentos excepcionales: primeros pasos para incluirlos en la escuela. Revista Colombiana de Educación, 1/79)., 423-444. https://doi.org/10.17227/rce num79-10040

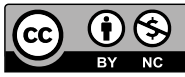

Recibido: 31/05/2019

Evaluado: 11/09/2019

Doctora en Educational Psichology, Gifted and Talent development de la Universidad de Connecticut. Consultora educativa independiente, Bogotá, Colombia. Correo electrónico: maria.caridad.garcia@gmail.com

** Magíster en educación. Consultora educativa independiente. Correo electrónico: juliana.iglesias@agmail.com 


\begin{abstract}
Resumen
En las últimas tres décadas, las políticas educativas y aproximaciones académicas a las capacidades y talentos excepcionales en Colombia han cambiado significativamente. Esto ha permitido orientar las decisiones de las instituciones educativas para atender a dicha población. En el 2015 se encuestaron 1731 instituciones educativas colombianas. Los resultados se usaron como base para el Documento de orientaciones técnicas, administrativas y pedagógicas para la atención educativa a estudiantes con capacidades y/o talentos excepcionales en el marco de la educación inclusiva, publicado por el Ministerio de Educación Nacional de Colombia. Algunas preguntas de la encuesta miden la comprensión de las instituciones sobre la excepcionalidad, pero en ese momento no fueron abordadas. Este artículo expone los resultados del análisis de dichas preguntas. Se identificaron tres tendencias para comprender la excepcionalidad: rendimiento, potencialidad y medición de inteligencia. Se observó que las aproximaciones tenían concordancia con algunas de las posturas de los documentos académicos y de política. Sin embargo, es posible que estas aproximaciones no sean conducentes a las políticas actuales de educación inclusiva. Es importante hacer un proceso de acompañamiento a las instituciones a fin de darles herramientas para construir las estrategias de reconocimiento y desarrollo de las capacidades y talentos excepcionales en concordancia con las políticas de educación inclusiva vigentes.
\end{abstract}

\section{Palabras clave}

Capacidades excepcionales; talentos; altas capacidades; inclusión; políticas educativas

\section{Keywords}

Gifted education; academically gifted; talent development; inclusion; educational policy

\begin{abstract}
Educational policies and academic approaches to exceptional skills and talents in Colombia have changed significantly in the last three decades. These changes have guided the decisions made by educational institutions to deal with that population.In 2015, 1731 Colombian institutions were surveyed. The results were the basis of the Documento de orientaciones técnicas, administrativas y pedagógicas para la atención educativa a estudiantes con capacidades y/o talentos excepcionales en el marco de la educación inclusiva [Technical, administrative, and pedagogical guidelines for the educational care of specially skilled and/or gifted students from an inclusive education perspective], published by the Colombian Ministry of National Education. Some questions in the survey measure institutional understanding of exceptionality, but they had not been addressed so far. In this paper, the authors analyze those questions. The analysis identifies three approaches to exceptionality: performance, potential, and intelligence measurement. Although these approaches coincide with policy and academic documents, they might not lead to current inclusive education policies. It is important to keep tracking the process with the institutions in order to provide them with tools that allow them to recognize and develop exceptional skills and talents, in agreement with current inclusive education policies.
\end{abstract}

\section{Resumo}

Nas últimas três décadas, as políticas educacionais e as abordagens acadêmicas para habilidades e talentos excepcionais na Colômbia mudaram significativamente. Isso permitiu orientar as decisões das instituições de ensino para servir a referida população. Em 2015, 1.731 instituições educacionais colombianas foram pesquisadas. Os resultados foram utilizados como base do Documento de Orientações Técnicas, Administrativas e Pedagógicas para atendimento educacional a estudantes com habilidades e / ou talentos excepcionais no âmbito da educação inclusiva, publicado pelo Ministério da Educação Nacional da Colômbia. Algumas perguntas da pesquisa questionaram o entendimento do excepcionalismo pelas instituições. Como na época não foram abordados, este artigo apresenta os resultados de análises secundárias realizadas no referido banco de dados. Para isso, foi utilizada uma análise de conteúdo com categorias dedutiva e indutiva das respostas das 1731 instituições. Como resultado, três tendências foram identificadas para entender a excepcionalidade: desempenho, potencialidade e medição de inteligência. As abordagens foram consideradas consistentes com algumas posições nos documentos acadêmicos e de políticas. No entanto, essas abordagens podem não ser favoráveis às atuais políticas de educação inclusiva. É importante realizar um processo de acompanhamento às instituições, a fim de fornecer ferramentas para a construção de estratégias de reconhecimento e desenvolvimento de capacidades e talentos excepcionais, de acordo com as políticas de educação inclusiva em vigor.

\section{Palavras-chave}

Capacidades excepcionais; talentos; alta capacidade; inclusão; políticas educacionais 


\section{Introducción}

Para algunos educadores y académicos, no tiene sentido hablar de capacidades y talentos excepcionales en el marco de la educación inclusiva. Entre estos se encuentran aquellos que plantean que la escuela no es el lugar más apto para responder a las necesidades de esta población (Matthews y Dai, 2014; Robinson y Clinkenbeard, 2008); y aquellos que definen su postura siguiendo el mito que asume que estos estudiantes manifestarán grandes desempeños sin la necesidad de apoyo de las instituciones y que, por tanto, no presentan problemas ni desafíos (Moon, 2009). No obstante, desde los años noventa y en función de las políticas -inicialmente de integración educativa y posteriormente de educación inclusiva- se ha repensado la pregunta de si es necesario excluir al estudiante con capacidades y talentos excepcionales de la escuela regular.

Autores como Renzulli y Reis (2014) plantean que lo que se requiere es un proceso de transformación total de la escuela, de manera que estudiantes con diversas potencialidades encuentren oportunidades educativas que permitan potenciar sus capacidades y talentos, transformándolos en producción creativa y experta. Esta propuesta, desde la perspectiva de estos autores, genera un enriquecimiento de toda la escuela que conduce a un incremento de la calidad educativa (Renzulli y Reis, 1994). En este sentido, se empieza a hablar de una escuela donde sus estudiantes desarrollan su potencial, no a pesar de ella, sino gracias a ella.

La Organización de las Naciones Unidas para la Educación, la Ciencia y la Cultura (Unesco), por su parte, al abordar la idea de la educación inclusiva y el enfoque diferencial, argumenta que

[...] cada niño tiene características, intereses, capacidades y necesidades que le son propias; si el derecho a la educación significa algo, se deben diseñar sistemas educativos y desarrollar los programas de modo que tengan en cuenta toda la gama de esas diferentes características y necesidades individuales. (Unesco, 1994, p. viiii).

En el 2015, en Colombia, siguiendo estos planteamientos dentro del contexto de la política educativa, se propuso que la comprensión del desarrollo de la excepcionalidad debía tomar como pilares fundamentales la educación inclusiva y el enfoque diferencial. Así se explicitó en el Documento de orientaciones técnicas, administrativas y pedagógicas para la atención educativa a estudiantes con capacidades y/o talentos excepcionales en el marco de la educación inclusiva del Ministerio de Educación Nacional (MEN). ${ }^{1}$

1 Su elaboración fue liderada por la Fundación FEs, la Facultad de Educación de la Pontificia Universidad Javeriana y el Ministerio de Educación Nacional, en el marco del Convenio 759-2015. 
Concretamente, se enunció que en el marco de una educación para todos es importante ofrecer a los estudiantes oportunidades de desarrollo que reconozcan sus características, fomenten sus potencialidades y permitan su participación como aprendices y ciudadanos (MEN, 2015). Además, se planteó que "no reconocer las diferencias tiene como consecuencia que muchos estudiantes no desarrollen sus capacidades pues encuentran barreras para el aprendizaje y su participación en la escuela" (p. 31).

Sin embargo, durante la elaboración del documento que consolidó esta idea dentro de la política, se hizo una encuesta en línea a las instituciones educativas para poder identificar las principales necesidades que tenían frente a la atención de poblaciones excepcionales y la forma como se aproximaban a ellas. ${ }^{2} \mathrm{Al}$ analizar los resultados, inicialmente se identificó que la mitad de las instituciones indicaron que sí abordaban la temática, pero solo la tercera parte de estas conocían los documentos de la política (MEN, 2015). Además, una tercera parte de las instituciones reportó que en el Proyecto Educativo Institucional (PEI) se hacía referencia explícita a esta población; en tanto que una quinta parte reportó haber recibido apoyo de las secretarías de Educación para la gestión de procesos de formación y atención, solo un 13 \% reportó tener articulación con otras instituciones para la oferta de mecanismos de atención a poblaciones con capacidades excepcionales. ${ }^{3}$ También, al "analizar la coherencia entre definiciones de 'capacidades o talentos excepcionales', los mecanismos de identificación y las estrategias de atención, se observa desarticulación entre los aspectos, lo cual puede indicar la falta de fundamentación, formación y apoyo en esta temática" (MEN, 2015, p. 22).

Estos resultados generaron la inquietud sobre cómo comprenden en realidad la excepcionalidad las instituciones educativas a la hora de reconocer e implementar acciones con esta población. Estas inquietudes dan pie al análisis de datos secundarios presentado en este artículo.

\section{Aproximación metodológica}

Para darle respuesta a este interrogante se retomó la base de datos de la encuesta y se examinaron, a partir de un análisis de contenido con categorías inductivas y deductivas, las preguntas relacionadas con la definición de las instituciones sobre la excepcionalidad y con las acciones emprendidas efectivamente en la institución educativa.

2 En esta encuesta participaron 1731 instituciones educativas del país, ubicadas en las 95 entidades territoriales certificadas en el país (MEN, 2015).

3 Tomado del Documento de recopilación de la revisión de antecedentes sobre la organización de la oferta y atención educativa a estudiantes con capacidades y talentos excepcionales publicado por la Fundación FEs, la Facultad de Educación de la Pontificia Universidad Javeriana y el MEN, en el marco del Convenio 759-2015. 
Primero se comenzó el proceso con una contextualización de las conceptualizaciones académicas y políticas acerca de las capacidades y talentos excepcionales para generar un marco de comprensión. Luego se analizaron las respuestas cerradas con el fin de hacer una caracterización general, para después entrar en las definiciones que dieron del término capacidades y talentos excepcionales.

En este punto se determinaron unas categorías y se identificaron las tendencias que emergieron de las respuestas. Posteriormente, se analizaron las acciones o inacciones reportadas por las instituciones en cada una de las tendencias, con lo cual se evidenciaron las dinámicas entre comprensión de la excepcionalidad y su efecto en la práctica.

\section{Aproximaciones académicas a las capacidades y talentos excepcionales}

En la actualidad, como múltiples autores han señalado, no hay un acuerdo frente a lo que son las capacidades y el talento excepcional (García-Cepero, 2015; Matthews y Dai, 2014; Robinson y Clinkenbeard, 2008) y de cómo debe abordarse desde el sistema educativo (Dai, 2018; García-Cepero, 2015).

En las últimas décadas diversos autores (entre ellos Sternberg, Renzulli y Dai) han construido taxonomías o mapeos que permiten mostrar los cambios en las concepciones sobre la excepcionalidad. Renzulli (2012) plantea dos concepciones de la excepcionalidad y su desarrollo: una tradicional, centrada en los resultados de pruebas de inteligencia y en las labores de la escuela tradicional; y otra contemporánea, centrada en el reconocimiento y la potenciación de la productividad creativa. Esta última se centra en el concepto de comportamientos excepcionales (gifted behavior), no en la excepcionalidad como un atributo estructural de la persona (Renzulli, 2012).

Kaufman y Sternberg (2008) plantean que las concepciones de excepcionalidad se han desarrollado por olas que, a pesar de evolucionar temporalmente, coexisten de manera sincrética en la actualidad. En la primera, el centro de las concepciones es la inteligencia como habilidad natural (en algunos casos innata y heredada). En la segunda ola, la visión monolítica es desafiada por los modelos de dominio específico que amplían la concepción de capacidades excepcionales y fundamentan la concepción de talento (Kaufman y Sternberg, 2008). En la tercera ola, Kaufman y Sternberg (2008) incluyen aquellos modelos que se aproximan a la comprensión de la excepcionalidad desde una perspectiva sistémica. Finalmente, los modelos de la cuarta ola son aquellos que comprenden desde una perspectiva de desarrollo el fenómeno de la excepcionalidad (Kaufman y Sternberg, 2008). 
Más recientemente David Yun Dai (Dai, 2010, 2018; Dai y Chen, 2013) ha analizado la evolución de las diversas concepciones de excepcionalidad y encuentra dos paradigmas fundamentales que se enfrentan en la actualidad: el esencialista y el desarrollista. El primero, que se aproxima al Ilamado por Renzulli (2012) tradicional y a los modelos de la primera y segunda ola, y algunos de la tercera en Kaufman y Stenberg (2008), y el desarrollista, corresponden en general a autores de la tercera y cuarta ola del modelo de Kaufman y Stenberg (2008).

Los contrastes de estas dos posturas, como se señaló en el Documento de orientaciones (MEN, 2015), hacen evidentes tensiones ontológicas. Por ejemplo, si el excepcional es o se desarrolla; si esa excepcionalidad está determinada por una habilidad general que se despliega en diferentes campos o si está determinada por habilidades y campos específicos. Además, se plantean tensiones epistemológicas, una de estas alrededor del reconocimiento de la excepcionalidad ya sea por el potencial del individuo enmarcado por pruebas de aptitudes, habilidades (o inteligencia), o por desempeño del individuo en actividades auténticas. Finalmente, plantea tensiones normativas que cuestionan sobre las metas que tiene el trabajo con dichas poblaciones.

Es en este contexto académico que se desarrolla el Documento de orientaciones del MEN del 2015, es el mismo contexto que rodea a las instituciones en el momento de realizar el sondeo.

\section{Antecedentes de la política de capacidades y talentos excepcionales en Colombia}

El tema de las capacidades y talentos excepcionales se introdujo en Colombia en la década de los ochenta en el ámbito de la educación privada, aunque solo a principios de los noventa comenzó a considerarse un asunto gubernamental, exactamente con la Constitución política de 1991 (Asamblea Nacional Constituyente, 1991) donde se definió que la educación de las personas con capacidades y talentos excepcionales era una de las obligaciones especiales del Estado (artículo 68). A partir de ese momento, se comenzó a configurar esa política pública y se emitieron documentos para orientar el proceso de educación de estos estudiantes.

Inicialmente no se estableció ninguna definición concreta del término excepcionalidad (Ley 115 de 1994, Resolución 2082 de 1996 y Ley 361 de 1997), pero esto cambió con los documentos de lineamientos de la política. La primera definición se presentó en el 2001 y la segunda en el 2016.

En el documento del 2001, Lineamientos generales de política para la atención de personas con talentos y/o capacidades excepcionales (MEN, 2001), se planteó que la excepcionalidad se podía caracterizar en dos 
tipos, los superdotados y los talentos, y se requería formas de educación diferente para cada grupo. Luego, se argumentó que

Superdotado es alguien que obtiene resultados fuera de lo común (en la mayoría de los casos por encima del $98 \%$ de la población) en pruebas desarrolladas para medir capacidad intelectual y predecir desempeño académico. Lo que define a un individuo superdotado es la posesión de tres conjuntos básicos de características estrechamente relacionadas, capacidad sobre el promedio, compromiso con la tarea y creatividad. (MEN, 2001, p. 5)

Para definir talento, lo hicieron en contraste de los superdotados y anotaron "su potencial no está relacionado con una capacidad académica general -como el superdotado- sino que está restringido a un dominio específico" (MEN, 2001, p. 5). Además, agregaron que los estudiantes con talento debían tener "un carácter decidido y una motivación bien dirigida hacia un campo" (MEN, 2001, p. 5).

Es importante señalar que en esta definición se siguen los planteamientos de Renzulli, pero simultáneamente y de manera sincrética se tienen en cuenta los planteamientos tradicionales de la excepcionalidad, que restringe esta propiedad al 2,5 \% de la población. Desde la postura de Renzulli, la prevalencia de la excepcionalidad puede ser de hasta del $20 \%$ de la población, pues las tres características que la definan (habilidad por sobre la media, compromiso con la tarea y creatividad) actúan de manera compensatoria y no eliminatoria.

En relación con la identificación se diferenció el proceso para cada caso. Para la superdotación se propuso la realización de pruebas de inteligencia, mientras que para los talentos se formuló primero que era necesario

[...] saber si ellos en realidad consideran su talento como un valor". Esto significaba que no solo debían tener "una alta competencia", sino que tenían que ser capaces de "trabajar sostenidamente y elaborar productos que, para su edad, superen con creces las expectativas para un individuo común". (MEN, 2001, pp. 5-6).

Las estrategias complementarias para esta identificación eran las entrevistas preliminares con padres y niños, y los cuestionarios a padres y profesores.

Con respecto a las acciones, en el documento de los lineamientos se propuso que no interfirieran con el curso normal de los procesos educativos, es decir, se ubicarían en la jornada contraria. Se mencionó la flexibilización curricular como una alternativa secundaria y sin profundizar. Del mismo modo, se dijo que el proceso debía hacerse en fases según los grados escolares. Una primera, denominada de exploración de la excepcionalidad, realizada en los grados de primaria para poder 
identificar a los estudiantes con esta característica. Una segunda, Ilamada de orientación, en la cual se buscaba que los alumnos detectados durante la básica secundaria se dirigieran a un dominio especifico según sus capacidades. La tercera y última fase, la educación de la excepcionalidad, ya no era responsabilidad de la educación básica, sino que se les atribuía a las universidades y a los centros de investigación, de producción artística y de trabajo social (MEN, 2001).

En el 2006 se comenzó a reconocer que existían diversas concepciones sobre la excepcionalidad, para después afirmar que en el caso colombiano era necesario sobrepasar las posturas que indicaban que la excepcionalidad era atributo de la persona (MEN, 2006). En sus términos, se requería comprender el concepto "como una necesidad educativa especial, fruto de una potencialidad en relación con el ambiente" y que "su atención depende de la transformación de la escuela y el entorno educativo" (MEN, 2006, p. 11).

Luego se realizó una propuesta de clasificación que abarcaba algunas de esas concepciones. En concreto se definieron cuatro tipologías: capacidades excepcionales globales, talentos excepcionales específicos, doble excepcionalidad e hiperestimulados.

Para abordar el proceso de identificación de esta población, se presentaron diferentes técnicas, entre las que se encontraban las no formales, las formales y la combinación de las dos. Aparte de eso retomaron algunas propuestas de evaluación realizadas desde la academia, con el fin de generar un panorama más amplio para que las secretarías de Educación y las instituciones educativas tuvieran conocimiento de las diferentes herramientas a las que podían recurrir para poder identificar a los estudiantes excepcionales.

El tema de las acciones se abordó con mayor amplitud y se presentaron dos opciones educativas: la de agrupamiento y la de integración escolar. Al sugerir el agrupamiento se estaba proponiendo no solo la opción de reunirlos en una institución o centro especializado, sino que incluían aulas especiales dentro de las escuelas y de escuelas satélites donde se agrupaban estos estudiantes en horarios extracurriculares o en jornada contraria, anexo a lo desarrollado en la escuela siguiendo el currículo oficial (MEN, 2006).

En cuanto a la integración, se refería al esfuerzo por la inclusión en las escuelas de los alumnos con NEE, buscando mejorar sus condiciones educativas. Dentro de esta línea se reconocieron apoyos o estrategias de atención para los estudiantes con excepcionalidad, entre los que se encontraba el enriquecimiento, la condensación del currículo, la aceleración, la figura de la tutoría y la educación en casa, o home schooling (MEN, 2006). 
Es importante señalar que, en el desarrollo de la historia de la política de atención a estudiantes excepcionales, hasta el momento de la realización del sondeo entran en juego tres hitos que en alguna medida pueden determinar lo que las instituciones educativas comprenden acerca de la excepcionalidad.

El primer hito es que existe un escenario común para la excepcionalidad y la discapacidad: la inclusión. Esta relación inicialmente se plantea de manera cercana, hasta el punto de presentar una estructura común de identificación y de acción para las dos poblaciones. Más aún, en el desarrollo normativo de la política de inclusión a mediados de los noventa, en la búsqueda por la consolidación de la descentralización institucional del país, se dictaminó que los entes territoriales que abordaran el tema de educación a través de las secretarías de Educación fueran los responsables de la atención a estas dos poblaciones (Resolución 2082 de 1996). En la normatividad que promocionó esta medida, se asumió que cada secretaría definiera cuáles instituciones educativas debían organizar aulas de apoyo especializadas para trabajar con la población de inclusión (discapacidad y excepcionalidad) orientadas por unidades de atención integral (UAI).

Un segundo hito consistió en que las aproximaciones estatales frente al concepto de excepcionalidad y la atención a dicha población sufrieron un proceso de ampliación, nutrido por elementos académicos. En este proceso, no solo se trasladó el término como característica a un tema de desarrollo, sino que se comenzó una tendencia por reconocer las diferentes aproximaciones conceptuales y educativas y darles validez, sin tener que casarse con una sola.

Un tercer hito es que a finales del siglo pasado se comenzó con un énfasis marcado por seleccionar las pruebas de inteligencia o conceptos de especialistas como el método para identificar a los estudiantes. Esa alternativa se presentó en los documentos de lineamientos, pero en el 2003, cuando se propuso reorganizar de nuevo la prestación del servicio educativo a la población de inclusión, se estableció que las secretarías de Educación definieran cuáles serían las instancias o las instituciones encargadas de determinar si los estudiantes manifestaban excepcionalidad o discapacidad, mediante una evaluación psicopedagógica y un diagnóstico interdisciplinario (Resolución 2565 del 2003, artículo 4).

Si bien el contexto político del campo de la excepcionalidad ha continuado su desarrollo, no se consideró pertinente incluir las modificaciones o ampliaciones posteriores a la realización del sondeo. Esto debido a que, en buena medida, las respuestas de las instituciones educativas al sondeo abordado en este artículo determinaron los contenidos y algunos aspectos de las aproximaciones del Documento de orientaciones técnicas, administrativas y pedagógicas para la atención educativa a estudiantes con 
capacidades y/o talentos excepcionales en el marco de la educación inclusiva, publicado en el 2015. Además, dieron pauta para las Orientaciones generales para la escuela y la familia en la atención educativa a estudiantes con capacidades y talentos excepcionales (2017) y para las Orientaciones para la transición educativa de los estudiantes con discapacidad y con capacidades o talentos excepcionales en la educación inicial, básica y media.

\section{Concepciones reportadas por las instituciones educativas sobre capacidades y talentos excepcionales}

En las respuestas de la encuesta se identificó que el 51 \% de las escuelas sí abordaban el asunto de las capacidades y talentos excepcionales e hicieron el ejercicio de presentar la definición institucional del tema. Un primer aspecto que indica que casi la mitad de las instituciones reconocen estar abordando la excepcionalidad, a pesar de que dista de lo que se espera desde una perspectiva inclusiva; es importante porque demuestra que el tema se ha hecho visible.

Por otra parte, se pudo observar que las definiciones coincidían con algunas de las posturas de los documentos académicos y de política. Exactamente el $88 \%$ de las instituciones que sí abordaban el tema, retomaban tres elementos utilizados por la academia y la política: el rendimiento, el potencial y la inteligencia. Sin embargo, se presentaron otras dos tendencias, una que relacionaba la excepcionalidad con discapacidad (10 \%) y otra que indicaba que era una característica general de toda la población escolar (2\%).

Aparte de estas estas cinco tendencias, se identificó otra. Entre los casos que indicaron que no trabajaban el tema de las capacidades y/o talentos excepcionales, se encontró que la mayoría de esas instituciones sí respondieron a la pregunta por la definición de la excepcionalidad. Esta información ayuda a comprender las razones de su postura.

A continuación se abordarán esas seis tendencias identificadas.

\section{Rendimiento}

Al mencionar el tema del rendimiento, se hizo una comparación de resultados utilizando términos como "desempeño por encima del promedio", "desempeño por encima de la media", "resultados superiores a los de sus pares", "desempeños superiores", "sus resultados sobresalen frente a sus compañeros" y "se destaca" entre otros. Estas comparaciones se realizaban sobre diferentes campos disciplinares o culturales, como 
por ejemplo campos académicos o aquellas que incluían otros como el campo deportivo, el artístico y el social. Estos últimos presentaban descripciones del rendimiento de manera más amplia y general. Además, es importante resaltar cómo, no necesariamente en las definiciones reportadas que involucran campos diversos, se encuentra la distinción que se hace en la academia o en la política entre las capacidades y el talento excepcionales (gifted frente a talented), que en general está determinada por la generalidad o la especificad del grupo de referencia y que aluden a campos culturales, disciplinares o sociales diferentes.

Con relación a los rendimientos académicos, las aproximaciones no solo mencionaban el término académico, ${ }^{4}$ sino que también incluían palabras como calificaciones, notas y respuestas en el aula como indicadores claros de las capacidades y talentos excepcionales. Es decir, en sus definiciones hacían referencia al ámbito académico escolar exclusivamente.

Unos ejemplos de esto son el caso 179, donde se afirmaba que eran "personas que sobresalen en una o más disciplinas del saber". El caso 29, donde se señalaba que estos estudiantes "demuestran un mayor desempeño frente a los otros de su mismo curso, de manera general en algunos aspectos generales". O el 527, "son aquellos estudiantes que se destacan en una o varias materias del plan de estudio y cuyo desarrollo académico es sobresaliente por encima de la media".

Sin embargo, en algunos casos se indicó que se requería que los resultados fueran constantes, permanentes en el tiempo o en una magnitud muy elevada. Para ilustrar este tipo de posiciones está el caso 189, donde se afirma que además de que estos estudiantes sobresalen académicamente, también la institución "ha observado estas capacidades durante largo tiempo" y agrega "sus capacidades los llevan a destacarse no solo en la institución sino también a nivel municipal y departamental".

Esta búsqueda en lo académico deja de lado a quienes tienen talentos excepcionales en áreas no "académicas" y afecta a aquellos estudiantes que manifiestan excepcionalidad en áreas muy específicas dentro de los campos académicos. A su vez, al adherir el ingrediente de la persistencia en el desempeño y al incrementar el impacto esperado, se genera un escenario de falsas expectativas que invisibiliza a la población de estudiantes con bajos logros y altas capacidades ("underachievers") o a los estudiantes con doble excepcionalidad. Esta última, recurrentemente ha sido reportada en la literatura como una población que es desatendida en el ámbito escolar (Conejeros, Cáceres y Oneto, 2010; Reis y McCoach,

4 En este caso empleamos el término académico para hacer referencia a las disciplinas del currículo básico escolar y excluyendo áreas como el deporte y el arte. Si bien este uso del término es poco preciso, lo mantuvimos por ser el término utilizado recurrentemente en los reportes de las instituciones. 
2002) y que se encuentra en riesgo particular en poblaciones vulneradas por condiciones socioeconómicas y culturales (Conejeros et ál., 2010; MEN, 2015).

Es así como concepciones centradas en el rendimiento en términos de notas escolares eliminan las posibilidades de reconocimiento y de acceso a oportunidades de desarrollo de estudiantes que, teniendo alto potencial, no logran manifestarlo, por diversos motivos, en los resultados esperados por la escuela. Esta situación se ha identificado en el contexto de los estudiantes chilenos, pero es aplicable al contexto colombiano:

[...] el grupo de estudiantes invisibles se transforma en una población escolar de mayor riesgo al incrementarse considerablemente las posibilidades de no desarrollar sus habilidades. Este hecho genera un cuestionamiento acerca de las políticas de identificación de estudiantes, utilizadas por los programas de talento chileno, las que están fuertemente cimentadas en la nominación docente. Se insinúa, por tanto, la importancia de generar rutas alternativas de nominación, como la autonominación. (García-Cepero et ál., 2012, p. 1338).

Por otra parte, se encontraron las definiciones que tomaban el rendimiento en términos generales, con descripciones como las siguientes:

“Eran estudiantes que se destacan en una o varias áreas académicas, artísticas o relacionales, siendo superior con relación a los pares y grado escolar". (Caso 150)

[o]

"[...] estudiantes que sobresalen de manera considerable con respecto a sus compañeros, en el campo académico, deportivo y/o cultural". (Caso 132).

Eran los casos en que se mencionaban distintas áreas de la acción humana donde se podía observar el desempeño de los estudiantes.

Dentro de este grupo de descripciones se presentaron algunas que plantearon que lo que se esperaba de estos estudiantes era que se destacaran solamente en un área determinada y era en esta que se observaban los desempeños sobresalientes. Se entendían las capacidades y los talentos de una manera particular unidireccional: "son estudiantes que poseen un alto potencial en un campo específico y desarrollan competencias superiores en este campo" (caso 161). Otro ejemplo enuncia: "estudiantes con capacidades superiores frente a otros estudiantes en un área específica" (caso 283).

Estas definiciones implican dos aspectos problemáticos. El primero es que no reconoce que con alguna frecuencia las capacidades excepcionales, se pueden manifestar en diversos campos, lo que se conoce como multipotencialidad (Achter, Lubinski y Benbow, 1996). En esa medida es positivo 
el paso que se da en el documento del 2015 (MEN, 2015), al reconocer que las capacidades y el talento excepcional constituyen un fenómeno heterogéneo y que agrupa una multiplicidad de perfiles. Esto debería llevar a que necesariamente las instituciones (lideradas por sus secretarias de Educación) propusieran múltiples estrategias de desarrollo y reconocimiento, para responder a la posible variabilidad de esta población.

El segundo desafío que presentan estas aproximaciones es que reconocen la excepcionalidad en la medida en que esta sea manifiesta. Sin embargo, si se parte de una postura desarrollista, la ausencia de manifestaciones no necesariamente implica la ausencia de excepcionalidad, pues esta puede encontrarse latente a la espera de condiciones apropiadas de desarrollo. Esto lleva a un círculo vicioso, pues solo cuando se ven manifestaciones se emprenden acciones de desarrollo, pero sin estas acciones es posible que las capacidades y el talento no se manifiesten espontáneamente. Por tanto, es importante reconocer, desde una postura desarrollista, que la excepcionalidad puede estar presente en diferentes estados, tanto como potencial o en algunos casos como desempeños, tal como enuncia en el documento de orientaciones publicado en el 2015, "la excepcionalidad se desarrolla, por tanto, es posible encontrarla como potencial o como desempeño, no es un atributo estable en la persona, sino que requiere de condiciones apropiadas para manifestarse" (MEN, 2015, p. 72).

\section{Potencial}

Ahora bien, frente a lo dicho, al analizar las respuestas es interesante encontrar que mencionaban que la excepcionalidad se relacionaba con las habilidades, potencialidades o actitudes de los estudiantes. Se encontró que eran descripciones que partían de un perfil implícito o caracterización de estos estudiantes en cuanto a cómo procesaban la información, pensaban y actuaban. A su vez, estas podían ser con o sin especificaciones.

Dentro de este espectro se encontraron definiciones que hablaban de "capacidades globales", "capacidades cognitivas", "capacidades intelectuales" diferentes a las de sus pares; de "ritmos académicos" o de "aprendizaje diferentes", de "inteligencia superior", de "habilidades particulares", de "potencialidades", sin profundizar en esos conceptos. Un ejemplo de este tipo de descripciones es la del caso 58: "son estudiantes que han desarrollado habilidades y/o destrezas en alguna actividad cultural, artística, deportiva, cognitiva o social".

También se presentaron otras respuestas, que propusieron más elementos, describiendo qué tipo de capacidades o habilidades poseen estos estudiantes, agregando la creatividad, el compromiso con la tarea y la solución de problemas. Para poder ilustrar esta gama de descripciones se 
comenzará con el caso 67, en el cual se detalla el tipo de habilidades que debían tener estos estudiantes, reportando "una disposición de habilidades neurolingüísticas y psicomotrices especiales que los hacen sobresalir en los procesos académicos" y se agrega que, a su vez, ostentaban "capacidades de metacognición". Otros mencionan además la creatividad, como el siguiente caso, en el que se argumenta que estos estudiantes se diferencian de los demás por "sus altos niveles comprensivos, de producción, creatividad, desempeño lingüístico, deducciones, curiosidad, interés por investigar entre otros" (caso 7).

Es necesario mencionar que estas respuestas Ilegan a un punto en el que comienzan a plantear actitudes y comportamientos como propias de los estudiantes excepcionales, lo que complejiza el perfil y las expectativas sobre esta población. Por ejemplo, en el caso 1327 se señalaba que los estudiantes excepcionales eran:

Personas con un alto potencial, general o restringido a un campo especifico de la actividad humana, para aprender y desarrollar competencias, que supera con creces al que se espera que tengan otras personas de su edad y en su medio, habilidades meta-cognitivas superiores y automaestría en una o varias áreas del saber. Se acompaña además de altos niveles de creatividad y de dedicación a las tareas que se emprenden.

Para cerrar esta ejemplificación, veamos el caso 95, que se extiende en su descripción y evidencia la complejidad de las expectativas que se tienen sobre esta población.

Les interesa y se les facilita comprender el funcionamiento y manipulación de mecanismos, aparatos y herramientas de tipo natural y artificial. O los que se interesan por participar en prácticas deportivas, consideran muy importante preservar su estado físico, siendo personas altamente competitivas. O quienes comprenden con mayor facilidad la mente (pensamientos, intereses, sentimientos, comportamientos) de los otros o de los grupos. Los que se interesan por entender y explicarse la realidad que los rodea, comprendiendo fácilmente ideas relacionadas con los fenómenos de la naturaleza y la sociedad o los individuos que tienen gran sensibilidad para ver en lo cotidiano, lo estético y lo artístico que otros no perciben o muestran también, grandes capacidades expresivas (corporales, escritas, orales). (Caso 95).

Estas definiciones son interesantes por cuanto reconocen la excepcionalidad como potencial; sin embargo, presentan un peligro: utilizan una serie de indicadores que las instituciones pueden entender como condiciones sine qua non para el reconocimiento de la excepcionalidad, y tienden a ser genéricas y corresponder a las formas en las que el potencial se puede observar en ciertos contextos (clase media alta, poblaciones 
caucásicas, con ambientes educativos enriquecidos). Es necesario dar un paso más adelante y contextualizar cómo se observa el potencial (y el desempeño) en los contextos particulares de la institución y del estudiante.

\section{Inteligencia}

Es preciso comenzar indicando que, si bien las concepciones presentadas en este apartado podrían integrarse dentro de las categorías anteriores, esto no se hizo porque este conjunto de respuestas hicieron énfasis en las pruebas de inteligencia de manera particular y no se definieron claramente como si fueran un indicador de desempeño o de potencial.

En estas respuestas que optaron por mencionar las pruebas de inteligencia como indicador de excepcionalidad, se observó que utilizaban distintas formas de denominarlas. Para algunos eran "pruebas para medir la capacidad intelectual", para otros "pruebas o test de inteligencia", "pruebas para medir el coeficiente intelectual" o "pruebas especializadas". Otros solo hablaban de "coeficiente intelectual" superior, mayor o igual a 140, por encima de la media.

Sin embargo, estas concepciones no evidencian una conceptualización clara sobre lo que es la inteligencia o sobre lo que ellos entienden que la prueba les permite reconocer. No obstante, esta aproximación no es la predominante, es la que con mucha frecuencia se encuentra en los modelos esencialistas de la excepcionalidad. Con esto no se quiere decir que esta aproximación es equivocada, pero entra en contradicción con las políticas vigentes, si se considera que las pruebas de inteligencia son el mejor o el único indicador de excepcionalidad. Esto se vuelve particularmente complicado en el caso del talento excepcional, pues los resultados de pruebas tradicionales de inteligencia no son indicadores oportunos o pertinentes de potencial o desempeño de talento en campos como las artes, el deporte o el liderazgo social.

Asimismo, es importante señalar que, como se ha reportado en investigaciones previas (MEN, 2015), las pruebas de inteligencia tienen limitaciones para reconocer el potencial intelectivo de los estudiantes cuando estos provienen de contextos diversos a aquellos para los cuales se crearon. Además, que "La excepcionalidad está determinada por factores contextuales, por tanto, debe comprenderse dentro de la cultura en la cual está inmerso el estudiante" (MEN, 2015, p. 72). Esto implica que los mecanismos o herramientas utilizados deben reconocer las formas en las que las capacidades se manifiestan en contextos específicos.

Por otra parte, tampoco se quiere decir que las pruebas de inteligencia no aporten indicadores oportunos para el reconocimiento de los estudiantes con capacidades excepcionales. Estas pueden ser un mecanismo que funciona de manera apropiada con algunas poblaciones, pero no con 
poblaciones diversas, o en condiciones de pobreza o vulneración. Es así como adquiere sentido lo que plantean autores como Renzulli (2012), quien incluye las pruebas de inteligencia, de aptitud y desempeño estandarizado como una de las rutas a través de las cuales un estudiante excepcional puede ser reconocido; pero que a su vez deben crearse múltiples mecanismos que permitan rastrear el potencial y los desempeños de los estudiantes desde los contextos particulares. Por ejemplo, las observaciones de los docentes y las oportunidades de reconocimiento ${ }^{5}$ que ellos puedan desarrollar desde su propia aula.

\section{Interferencia del concepto discapacidad}

Dentro de este estudio se encontró involucrado en las definiciones de excepcionalidad el término discapacidad (83 casos). Lo interesante es que se identificaron dos tendencias en las respuestas, una proponía que las capacidades y talentos excepcionales hacían referencia a una discapacidad: "son personas que tienen una discapacidad física o tienen problema de aprendizaje" (caso 1249). Otro es el caso 41, en el que se afirma que eran "Ios niños con dificultades de aprendizaje con síndrome de autismo leve, síndrome de Down leve, síndrome de Asperger", o el 102 que indicaba que era "cuando un estudiante tiene discapacidad mental o física o sordomudo" (caso 102).

Al explorar las respuestas de este grupo se encontró que la relación de conceptos se daba en algunos casos a través del tema de lo excepcional, entendido como una forma de interpretar la diferencia desde una aproximación positiva. Es decir, comprender que los estudiantes con discapacidad son seres excepcionales o especiales, que pueden alcanzar metas que inicialmente no se esperaba. Un ejemplo de estas respuestas está en el caso 74, donde se indica que estos son "niños que anteriormente fueron declarados limitados por su enfermedad genética, pero hoy día se les da un trato normal obteniendo de ellos logros que anteriormente no se esperaban".

En el segundo grupo, donde se hablaba de discapacidad y excepcionalidad de manera diferencial, se observaron dos tendencias de respuestas. En la primera, una donde estas eran representadas por unos estudiantes y luego agregaban que algunos estudiantes con discapacidad podían presentar excepcionalidad. La otra, presentó una diferenciación total entre discapacidad y excepcionalidad, mostrándolas como dos poblaciones distintas pero que estaban cobijadas o conectadas por la inclusión o por ser personas COn NEE.

5 En este punto no nos referimos a los exámenes escolares, sino a actividades que los educadores pueden hacer como parte del proceso educativo, por ejemplo, actividades de enriquecimiento triádico (Renzulli y Reis, 2014). 
Para ilustrar mejor la primera tendencia, se presenta una de las respuestas:

Personas con capacidades excepcionales - es el estudiante con una capacidad global, que obtiene resultados muy altos en pruebas para medir la capacidad intelectual y los conocimientos generales.

Persona con talento excepcional - el potencial de este estudiante se dirige a un área específica de la actividad humana, como las ciencias, las matemáticas, las artes o el deporte, entre otras. También puede presentarse capacidades excepcionales en personas con discapacidad. (Caso 1348).

Este conjunto de aproximaciones se relaciona con el concepto de doble excepcionalidad, que también estaba reconocido en el ámbito de la política, como se describió. En la mayoría de los casos se hizo solo la mención sencilla preliminar, pero en otros se utilizaba una concepción particular de esta aproximación, que se alejaba del acercamiento académico porque se mencionaba que era una posibilidad de todos los estudiantes con discapacidad en general, sin importar su condición, como el síndrome de Down o dificultades en el aprendizaje.

En la segunda tendencia, aquella que conseguía caracterizar la población con discapacidad y la población excepcional de manera independiente, como se reporta en el caso 1260, se hace referencia a

Estudiantes que presentan diferencias físicas y/o en su capacidad de aprendizaje que difieren significativamente de la media (por encima o por debajo) y que son tan amplias que requieren de la puesta en marcha de programas educativos especiales. El término estudiantes excepcionales incluye tanto a aquellos que experimentan problemas de aprendizaje, como a los que presentan una respuesta muy superior a la media, que requieren de apoyos especiales que les ayude a desarrollar todo su potencial. Por tanto, este término hace referencia a los estudiantes que poseen problemas de aprendizaje, problemas de conducta, discapacidades físicas, deficiencias sensoriales y a los estudiantes superdotados. (Caso 1260).

En esta tendencia aparentemente se equipara el término excepcionalidad con necesidades educativas especiales, lo cual tiene sentido si se observa cómo en la política educativa los estudiantes excepcionales y aquellos en condición de discapacidad compartían la misma legislación hasta el 2017. Es decir, en su historia las dos poblaciones han sido desarrolladas bajo la misma sombrilla política.

Pero esta concepción de la política de inclusión en algunos casos deja una alternativa que consiste en aplicar el procedimiento y las rutas que se aplican para la población con discapacidad a la población con 
capacidades o talentos excepcionales. De esta manera se pone énfasis en los diagnósticos realizados por especialistas de la salud como la vía de identificación, con lo cual se le deja la responsabilidad a alguien externo al colegio. Esta postura en algún momento se consideró viable en el desarrollo normativo de la inclusión (Resolución 2082 de 1996 y Resolución 2565 del 2003). Sin embargo, esta aproximación perdería vigencia si la excepcionalidad se concibe desde una perspectiva pedagógica, como se presenta en el documento de orientaciones del 2015, donde la principal labor no es identificar quién "es" o quién "no es" excepcional, pregunta fundamentada en posturas especialistas, sino "bajo qué condiciones se manifiesta la excepcionalidad".

\section{Excepcionalidad sin excepcionales}

Una aproximación que se presentó en solo el $2 \%$ de las instituciones es aquella que indicaba que la excepcionalidad era una característica general de toda la población escolar. Este enfoque se acerca a los planteamientos de Borland (2005) en Gifted education without gifted children. Es interesante observar cómo en algunas instituciones el abordaje de la excepcionalidad se deja de implementar en función de unos principios de igualdad, que podrían estar sacrificando las necesidades particulares de las poblaciones excepcionales, pues si bien todos los estudiantes son iguales en derechos y dignidad, no son iguales en las necesidades que tienen para presentar un óptimo desarrollo.

\section{Las instituciones que reportan no abordar el tema de la excepcionalidad}

Inicialmente se encontró que las instituciones educativas que indicaron no abordar el tema no lo hacían por desconocimiento total de este, ya que menos del $2 \%$ de las escuelas señalaron que no tenían una definición. La mayoría reconocieron una caracterización de este tipo de estudiantes con elementos comunes como desempeños superiores que sus pares o sobresalientes, con coeficiente intelectual superior a 130, aprendizajes acelerados, mayores habilidades para manejar el conocimiento entre otros.

Puntualmente, se identificó que es producto de una concepción particular de la excepcionalidad, en la que se describen como algo natural que puede presentarse en las escuelas, pero no que no requiere acciones particulares por parte de la institución. Una realidad subyacente que no exige acción alguna por parte del sistema. Por tanto, existe una creencia subyacente de que esta población se encuentra en la escuela, pero no requiere un abordaje por parte de las instituciones. Además, que se realza positivamente sobre los otros estudiantes y eso parece ser suficiente. 
Esta aproximación en alguna medida puede estar fundamentada en posturas esencialistas, donde se plantea que la excepcionalidad "es" una condición estructural de la persona y que se manifestará "a pesar" de las condiciones ambientales y de manera espontánea. En términos de Marulanda-Páez y García-Cepero (2018), está determinada por las leyes de la supervivencia del más fuerte. Esta postura vuelve a invisibilizar a esta población y no reconoce que cuando el estudiante no puede transformar su potencial en desempeño, la escuela, la familia y la sociedad tienen responsabilidad en este fracaso. El problema de la excepcionalidad está centrado, pues, en un desajuste entre lo que los estudiantes requieren para poder convertirse en la mejor versión de ellos mismos y lo que la escuela y la sociedad les están ofreciendo.

\section{A modo de reflexión final}

Dado que existen aproximaciones en las instituciones tan diversas, es importante comprender los abordajes particulares de cada una y comprender cómo ellas llegan a construir los conceptos que guían las acciones (o inacciones) de reconocimiento y la construcción de oportunidades de desarrollo de poblaciones excepcionales (y de cualquier otra población). Es importante entender el impacto que ha tenido para el campo de la excepcionalidad el compartir un proceso histórico con el de la discapacidad. Sin embargo, es necesario diferenciar las aproximaciones, de manera que los sesgos clínicos no se constituyan en barreras para el desarrollo de oportunidades de reconocimiento desde una apuesta pedagógica.

Por otra parte, es plausible explicar el sincretismo de las concepciones preponderantes en las instituciones por una yuxtaposición de políticas y tendencias teóricas que, al coexistir al menos virtualmente a pesar de corresponder a momentos históricos distintos, generan aproximaciones que pueden ser contradictorias y confusas.

A partir de este ejercicio no es posible saber si el documento de orientaciones publicado en el 2015 permite resolver algunas de las contradicciones, vacíos o falencias presentes en las aproximaciones reportadas en el sondeo realizado en el 2015. Pero sí es claro que es indispensable una política de difusión fuerte y de formación en la temática, para hacer propuestas sólidas que empoderen a las instituciones y a las secretarías de Educación para construir el andamiaje necesario que permita ofrecer oportunidades de reconocimiento y de desarrollo oportunas y pertinentes para los estudiantes con capacidades y/o talentos excepcionales.

Finalmente, y a modo de cierre como plantea García-Cepero (2015)

[...] en la actualidad, y dado el marco de las políticas de inclusión, el problema no es que dicha población [estudiantes con capacidades y 
talentos excepcionales] se encuentre excluida de la escuela, muchos de ellos se encuentran en ella, pero quien no está incluido en la escuela es el talento. La escuela ha estado tan acostumbrada a ser un espacio de remediación, que pareciera que el capitalizar en el potencial de sus estudiantes, no es parte de su misión ni su visión. Tiende a concentrarse tanto en lograr los objetivos mínimos, que ignora que más allá de ellos hay muchísimo camino por recorrer. La escuela y sus docentes no son conscientes de rol como catalizador en la emergencia del talento de los estudiantes. (P. 68).

\section{Referencias}

Achter, J. A., Lubinski, D. y Benbow, C. P. (1996). Multipotentiality among the intellectually gifted: "It was never there and already it's vanishing.". Psychology, Journal of Counseling, 43(1), 65.

Borland, J. H. (2005). Gifted education without gifted children. Conceptions of giftedness. Cambridge University Press.

Asamblea Nacional Constituyente (1991). Constitución Política de Colombia.

Conejeros, M. L., Cáceres, P. y Oneto, P. (2010). Informe final. La desatención educativa diferencial: consecuencias socio-emocionales en adolescentes con altas capacidades. Santiago de Chile: Fonide, Ministerio de Educación de Chile.

Constitución política colombiana. (1991). Asamblea Nacional Constituyente, Bogotá, Colombia, 6 de julio de 1991.

Dai, D. (2010). The nature and nurture of giftedness: A new framework for understanding gifted education. Nueva York: Teachers College Press.

Dai, D. y Chen, F. (2013). Paradigms of gifted education: A guide for theory-based, practice-focused research. Wako: Prufrock Press.

Dai, D. Y. (2018). A history of giftedness: Paradigms and paradoxes. En Handbook of Giftedness in Children (pp. 1-14). Cham Suiza: Springer.

García-Cepero, M. (2015). El sentido de una educación para el desarrollo del talento. Revista Javeriana, 151(817), 66-70.

García-Cepero, M. C., Proestakis, A. N., Lillo Olivares, A., Muñoz M. E. M., López Valladares, C. y Guzmán Garay, M. I. (2012). Caracterización de estudiantes desde sus potencialidades y talentos académicos en la región de Antofagasta, Chile. Universitas Psychologica, 11(4), 1327 1340.

Kaufman, S. y Sternberg, R. (2008). Conceptions of giftedness. En S. Pfeiffer (Ed.). Handbook of giftedness in children (pp. 71-91). Tallahassee, FL: Springer. 
Matthews, D. J. y Dai, D. Y. (2014). Gifted education: Changing conceptions, emphases and practice. International Studies in Sociology of Education, 24(4), 335-353.

Marulanda-Páez, E. y García-Cepero, M. C. (2018). La educación inclusiva en la Universidad. Más allá de la supervivencia del más fuerte. En Pedagogía, formación e innovación: Reflexiones de maestros para maestros. Colección 4C. Bogotá: Facultad de Educación, Pontificia Universidad Javeriana.

Ministerio de Educación Nacional de Colombia (1996). Decreto 2082, por el cual se reglamenta la atención educativa para personas con limitaciones o con capacidades o talentos excepcionales.

Ministerio de Educación Nacional de Colombia (2001). Lineamientos generales de política para la atención de personas con talentos y/o capacidades excepcionales. Bogotá.

Ministerio de Educación Nacional de Colombia (2003). Resolución 2565. Por la cual se establecen parámetros y criterios para la prestación del servicio educativo a la población con necesidades educativas.

Ministerio de Educación Nacional de Colombia (2006). Orientaciones para la atención educativa a estudiantes con capacidades y talentos excepcionales. Bogotá.

Ministerio de Educación Nacional (2015). Documento de orientaciones técnicas, administrativas y pedagógicas para la atención educativa a estudiantes con capacidades y/o talentos excepcionales en el marco de la educación inclusiva. Bogotá.

Moon, S. M. (2009). Myth 15: High-ability students don't face problems and challenges. Gifted Child Quarterly, 53(4), 274-276.

Organización de las Naciones Unidas para la Educación, la Ciencia y la Cultura-Unesco (1994). Marco de Acción de la Conferencia Mundial sobre Necesidades Educativas Especiales: Acceso y Calidad. Salamanca.

Reis, S. M. y McCoach, D. B. (2002). Underachievement in gifted students. The social and emotional development of gifted children: What do we know.

Renzulli, J. S. (2012). Reexamining the role of gifted education and talent development for the 21st century: A four-part theoretical approach. Gifted Child Quarterly, 56 (3), 150-159.

Renzulli, J. S. y Reis, S. M. (1994). Research related to the schoolwide enrichment triad Model. Gifted Child Quarterly, 38(1), 7-20.

Renzulli, J. S. y Reis, S. M. (2014). The schoo/wide enrichment model: A how-to guide for talent development. Waco: Prufrock Press.

Robinson, A. y Clinkenbeard, P. R. (2008). History of giftedness: Perspectives from the past presage modern scholarship. In Handbook of giftedness in children (pp. 13-31). Springer, Boston, MA. 